\title{
Change of coronary artery indices according to coronary dominance pattern in early childhood
}

Yoon Jin Lee, MD, Kyoung Soo Park, MD, Hong Ryang Kil, MD, PhD

Department of Pediatrics, College of Medicine, Chungnam National University, Daejeon, Korea

Purpose: Coronary arterial lesion assessment in children can be difficult, depending on the coronary dominance pattern. Although it is easier to determine coronary dominance with echocardiography in children than in adults, it is still difficult. This study aimed to examine the coronary dominance pattern according to the objective coronary artery (CA) indices.

Methods: The CA diameter, aortic valve annulus, and abdominal aorta of 69 children without any cardiovascular disease were measured with cross-sectional echocardiography at Chungnam National University Hospital. To evaluate the coronary dominance pattern, echocardiography was primarily used; additionally, coronary computed tomographic angiography or coronary angiography (CAG). Coronary dominance was determined according to the status of the CA that gives rise to the posterior descending artery.

Results: The mean age was $4.02 \pm 2.78$ years, and the mean body surface area (BSA) was $0.70 \pm 0.22$ $\mathrm{m}^{2}$. Right dominance was present in $78 \%$ and left in $22 \%$ of the subjects. In those with left dominance, the CA to aortic valve annulus diameter ratio was $0.125 \pm 0.021$ in the right coronary artery (RCA) and $0.255 \pm 0.032$ in the left coronary artery (LCA). In those with right dominance, the corresponding ratio was $0.168 \pm 0.028$ in the RCA and $0.216 \pm 0.030$ in the $L C A(P<0.05)$. Significant differences were also found in the diametric ratios of the CA to BSA and abdominal aorta $(P<0.05)$.

Conclusion: The CA indices showed significant difference according to the coronary dominance pattern in early childhood. It is possible to indirectly determine the coronary dominance pattern with the CA indices in children using echocardiography. The accuracy of coronary artery lesion diagnosis can be improved by taking coronary dominance into account.

Key words: Kawasaki disease, Coronary artery

\section{Introduction}

Variants of coronary artery (CA) anatomy are encountered in several types of congenital heart disease. Kawasaki disease (KD) is the most common cause of acquired heart disease in children living in developed countries. The diagnosis relies on clinical observation, and, in most cases, CA dilation or aneurysms are assessed using echocardiography. ${ }^{1)}$ In adults, CA anatomy and anomalies can be accurately evaluated using noninvasive imaging of the CA with coronary computed tomographic angiography (CCTA). However, the use of CCTA is limited in children. Although normalization of the CA dimension for body surface area (BSA) as $z$ scores based on regression equations allows standardization as a continuous measure in the Japanese Circulation Society (JCS) and American Heart Association guideline, the assessment of a coronary artery lesion (CAL) can be difficult depending on the pattern of coronary dominance. ${ }^{2,3)}$ Although a study of the coronary-aorta index (CA-to-aortic-annulus ratio) or normal CA dimensions established reference ranges for CA in children, it did not take
Corresponding author: Hong Ryang Kil, MD

Department of Pediatrics, Chungnam National University Hospital, Chungnam National University School of Medicine, 282 Munhwa-ro, Jung-gu, Daejeon 35015, Korea

Tel: $+82-42-280-7251$

Fax: +82-42-255-3158

E-mail: gilhong@cnu.ac.kr

https://orcid.org/0000-0003-4925-8240

Received: 1 October, 2018

Revised: 9 November, 2018

Accepted: 22 November, 2018
Copyright (C) 2019 by The Korean Pediatric Society

This is an open-access article distributed under the terms of the Creative Commons Attribution NonCommercial License (http://creativecommons.org/ licenses/by-nc/4.0/) which permits unrestricted noncommercial use, distribution, and reproduction in any medium, provided the original work is properly cited. 
coronary dominance into account. ${ }^{4,5}$ No criterion exists with respect to variations in the inner diameters of the right coronary artery (RCA) and left coronary artery (LCA) according to coronary dominance in the literature, thus causing considerable difficulty in evaluating CAL. Additionally, it is difficult to determine coronary dominance with echocardiography in adults as well as children. If CAL is evaluated without taking coronary dominance into consideration, a normal CA could be determined as abnormal. If a change of CA indices according to known coronary dominance is determined, the accuracy of CAL diagnosis can be improved by taking coronary dominance into account. Accordingly, the authors conducted the present study to examine the change of $\mathrm{CA}$ indices according to the pattern of coronary dominance.

\section{Materials and methods}

\section{Subjects}

The subjects of the study were 69 children ( 32 boys and 37 girls) who were referred to the Department of Pediatrics at Chungnam National University Hospital between June 2017 and June 2018. Children included in the study were subjected to full clinical and echocardiographic examinations before enrollment. Criteria for children included in the study were that they were between 3 months and 13 years of age, that they had structurally normal hearts with normal clinical and echocardiography examinations, and that echocardiography was to be performed for one of the following reasons: evaluation of asymptomatic cardiac murmurs, musculoskeletal chest pain, or a suspected abnormal chest X-ray. In 5 of 69 subjects, coronary angiography (CAG) or CCTG was performed for the evaluation of uncertain CALs or anomalies.

\section{Procedures}

The subjects were examined using an ultrasound system (Vivid 7 imaging system, GE, Chicago, IL, USA) connected to standard transthoracic transducers, in the supine or lateral decubitus position. For each subject, the highest frequency transducer with adequate penetrance was used. All standard and modified apical, parasternal, and subcostal views were used to follow the course of the left main coronary artery, left anterior descending coronary artery, left circumflex coronary artery (LCX), and RCA, from the start of each artery and distally as far as possible using a previously published technique. ${ }^{6}$ The maximal internal luminal diameters of the right and left coronary arteries during diastole were measured at 5-10 $\mathrm{mm}$ distal to their origins from the aorta. The diameter of the aortic annulus was measured in standard parasternal long axis view, at end systole, from hinge point to hinge point of the aortic cusps. To assess the pattern of coronary dominance, the origin of the posterior descending artery (PDA) was examined on apical 4-chamber view, subcostal atrial long-axis view, or precordial long-axis view. Left or right coronary dominance was determined depending on whether the PDA originated from the LCX or the RCA ${ }^{3,4)}$ Only subjects whose pattern of coronary dominance was confirmed on either 2-dimensional or Doppler echocardiographic evaluation were included in this study. Patients with a balanced CA system were excluded from the analysis, because of the low number of subjects in this group and the difficulty in assessment with echocardiography alone. The coronary indices, aortic valve annulus, and descending aorta were checked examined according to the pattern of coronary dominance. The ratio of the RCA to the LCA in left coronary dominance in the present study was analyzed. The echocardiographic study was done by one cardiologist. The previous studies on CA indices were compared to the present study. ${ }^{4,5)}$ The Institutional Review Board (IRB) of Chungnam National University Hospital approved this study (2018-10-022). The requirement for informed patient consent was waived by the IRB.

\section{Statistical analysis}

Statistical analysis was performed using IBM SPSS Statistics ver. 23.0 (IBM Co., Armonk, NY, USA). All data are presented as means and standard deviations. The CA indices were visualized separately for each pattern of coronary dominance using Sigma plot version 12.0 (Merck KGaA, Darmstadt, Germany). An independent paired $t$ test was performed to compare the CA indices in each coronary dominance pattern, and an unpaired $t$ test was performed to compare the findings of the present study with those of previous studies. Statistical significance was determined at $P<0.05$.

\section{Results}

\section{Demographic characteristics of the study group}

The mean age of the subjects was $4.02 \pm 2.78$ years ( 3 months -13 years), and the sex ratio was 0.86:1 ( 32 boys and 37 girls). The mean weight was $17.79 \pm 7.75 \mathrm{~kg}(7.8-50 \mathrm{~kg})$, and the mean BSA was $0.70 \pm 0.22 \mathrm{~m}^{2}\left(0.34-1.47 \mathrm{~m}^{2}\right)$. Age, body weight, and BSA were not significantly different between the patterns of coronary dominance $(P>0.05)$. Of the total number of subjects, $21.7 \%$ (15 of 69) were determined to have left coronary dominance and 78.3\% (54 of 69)

Table 1. Demographic characteristics of the study group

\begin{tabular}{lccc}
\hline Characteristic & $\begin{array}{c}\text { Total } \\
(\mathrm{n}=69)\end{array}$ & $\begin{array}{c}\text { Left dominant pattern } \\
(\mathrm{n}=15)\end{array}$ & $\begin{array}{c}\text { Right dominant pattern } \\
(\mathrm{n}=54)\end{array}$ \\
\hline Age $(\mathrm{yr})$ & $4.02 \pm 2.78$ & $4.01 \pm 3.18$ & $4.02 \pm 2.69$ \\
Sex, male:female & $32: 37$ & $11: 4$ & $21: 33$ \\
BW $(\mathrm{kg})$ & $17.79 \pm 7.75$ & $15.74 \pm 4.86$ & $18.37 \pm 8.34$ \\
BSA $\left(\mathrm{m}^{2}\right)$ & $0.70 \pm 0.22$ & $0.65 \pm 0.17$ & $0.71 \pm 0.23$ \\
\hline
\end{tabular}

Values are presented as mean \pm standard deviation or number.

$B W$, body weight; BSA, body surface area.

$P>0.05$. 
were determined to have right coronary dominance (Table 1).

\section{Comparison with previous study findings according to the pattern of coronary dominance}

A significant difference was found between the study by Tan et al. ${ }^{5)}$ and the present study in the diameter ratio of the CA to the aortic valve annulus, both in left and right coronary dominance $(P<0.05)$. The ratio of the RCA to the LCA in left coronary dominance in the present study was significantly different from that reported by Choi et al. ${ }^{4)}$; however, the corresponding ratio in right coronary dominance was not significantly different between the 2 studies $(P>$ 0.05) (Table 2). Choi et al. ${ }^{4)}$ and Tan et al. ${ }^{5}$ did not consider coronary dominance

\section{Analysis of $\mathrm{CA}$ indices according to the pattern of coronary dominance}

In left coronary dominance, the diameter ratio of the CA to the BSA was $0.509 \pm 0.134$ in the LCA and $0.246 \pm 0.053$ in the RCA, whereas the ratio in right coronary dominance was $0.409 \pm 0.099$ in the LCA and 0.316 \pm 0.069 in the RCA, showing significant differences between the LCA and RCA ratios to the BSA in both coronary dominance patterns $(P<0.05)$. Furthermore, the diameter ratios of the CA to the aortic valve annulus and the descending aorta were significantly different in both coronary dominance patterns $(P<0.05)$

Table 2. Coronary artery indices according to the dominant coronary arterial pattern: comparison with the studies of Choi et al. ${ }^{4)}$ and Tan et al. $^{5)}$

\begin{tabular}{lcccc}
\hline Variable & $\begin{array}{c}\text { Left dominant } \\
(n=15)\end{array}$ & $\begin{array}{c}\text { Right dominant } \\
(n=54)\end{array}$ & $\begin{array}{c}\text { Tan et al. } \\
(n=390)\end{array}$ & $\begin{array}{c}\text { Choi et al. } \\
(n=83)\end{array}$ \\
\hline LCA/AoA & $0.255 \pm 0.032$ & $0.216 \pm 0.030$ & $\mathbf{0 . 1 5} \pm 0.02$ & - \\
RCA/AoA & $0.125 \pm 0.028$ & $0.202 \pm 0.027$ & $\mathbf{0 . 1 3} \pm 0.02$ & - \\
RCA/LCA & $0.495 \pm 0.082$ & $0.785 \pm 0.118$ & - & $\mathbf{0 . 7 9 0} \pm 0.150$
\end{tabular}

Values are presented as mean \pm standard deviation.

LCA, left coronary artery; RCA, right coronary artery; AoA, aortic annulus; LCA AoA, left and right dominant vs. Tan et al.; RCA/AoA, left and right dominant vs.

Tan et al.; RCA/LCA, left dominant vs. Choi et al.

${ }^{*}$ Boldface indicates a statistically significant difference with $P<0.05$.

Table 3. Difference in the coronary arterial indices according to dominant coronary artery pattern

\begin{tabular}{lccr}
\hline Pattern & Left dominant pattern & Right dominant pattern & $P$ value \\
\hline LCA/BSA & $0.509 \pm 0.134$ & $0.409 \pm 0.099$ & 0.002 \\
RCA/BSA & $0.246 \pm 0.053$ & $0.316 \pm 0.069$ & $<0.001$ \\
LCA/AoA & $0.255 \pm 0.032$ & $0.216 \pm 0.030$ & $<0.001$ \\
RCA/AoA & $0.125 \pm 0.028$ & $0.202 \pm 0.027$ & $<0.001$ \\
LCA/dAo & $0.358 \pm 0.048$ & $0.299 \pm 0.041$ & $<0.001$ \\
RCA/dAo & $0.176 \pm 0.033$ & $0.233 \pm 0.034$ & $<0.001$ \\
\hline
\end{tabular}

Values are presented as mean \pm standard deviation.

LCA, left coronary artery; RCA, right coronary artery; AoA, aortic annulus; BSA, body surface area; $\mathrm{dAo}$, descending aorta.

Boldface indicates a statistically significant difference with $P<0.05$.
(Table 3).

\section{Discussion}

In the present study, right dominance was present in 78\% and left in 22\% of the subjects. Significant differences were found in the diametric ratios of CA to aortic valve annulus, BSA, and abdominal aorta according to coronary dominance pattern in early childhood.

Although CAG and CCTA are sometimes performed to obtain clear information about the CA (such as CA anatomy and extent of stenosis, obstruction, or aneurysm), their use in children are limited. Therefore, echocardiography is the primary imaging modality for cardiac assessment because it is noninvasive and has high sensitivity and specificity for the detection of abnormalities of the CA segments. ${ }^{7,8)}$ To diagnose CALs, the diagnostic criteria of the JCS KD committee proposed in 2013 are used. In the criteria, the presence or absence of CALs is determined on the basis of age-specific absolute values of the CA or the ratio to the caliber of a surrounding artery. ${ }^{2)}$ Such diagnostic criteria are inadequate in that age grouping is arbitrary, weight and height are not taken into account, and details on the measurement method are lacking. Normalization of the CA dimension for the BSA as $z$ scores (standard deviation units from the mean) based on regression equations allows standardization as a continuous measure and allows comparisons across time and populations. ${ }^{9,10)}$ However, even if CA caliber values normalized with age or BSA are applied, variations in the RCA and LCA according to coronary dominance can make it difficult to assess CALs. With antegrade-directed CA flow by transthoracic echocardiography, the PDA and the promixal and middle segments of the RCA were entirely seen in 54\%, 40\%, and 28\%, respectively, in adult subjects. Visualization of the complete RCA/PDA was negatively related to patients' body mass index in regression analyses. ${ }^{11)}$ Although it is easier to determine coronary dominance with echocardiography in children than in adults, difficulty still exists. In the present study, the authors were able to confirm the origin of the PDA in only about one-half of the sample. Therefore, if echocardiography is performed in children owing to limitations in using CAG and CCTA in clinical practice, indirect assessment of coronary dominance using CA indices is more useful than direct assessment of the precise distribution of the PDA.

The artery that supplies the PDA and a posterolateral branch determines the pattern of coronary dominance. Therefore, 3 situations are possible with normal variation: right dominance (approximately 70\%-80\% of the cases; supply from the RCA), left dominance (5\%-10\%; supply from the left circumflex coronary artery), and a balanced CA system (10\%-20\%). ${ }^{12-15)}$ To determine the pattern of coronary dominance in the present study, echocardiographies were performed on most subjects. Additionally, CAG and CCTA were performed on some subjects. Consequently, $22 \%$ of the subjects 
showed left coronary dominance and 78\% showed right coronary dominance. The relatively higher proportion of left coronary dominance $(22 \%)$ in the present study can be explained by the subjects' developmental stages (i.e., childhood) and the possibility that subjects with a balanced CA system were included in the group with left coronary dominance. The relatively low prevalence of left dominance in the general population and the decreasing prevalence of a left-dominant or balanced-dominance coronary system with age have raised the question of whether left dominance may represent a less well-balanced circulation with more myocardium at risk in acute coronary syndromes. ${ }^{15)}$ In a recent prospective study of 1,425 adult patients referred for CCTA, nonfatal MI and all-cause mortality were increased (hazard ratio, 3.15 ) in patients with left dominance during a 2-year follow-up period. ${ }^{16)}$

The diameter ratio of the LCA to the RCA in patients with left coronary dominance was approximately 1.62 , and this finding was similar to that reported by Dodge et al. ${ }^{17)}$ Hence, if the diameter ratio of the LCA to the RCA is $<1.62$ on echocardiography, coronary dominance can be inferred to be on the left side, even when the distribution of arteries on the left ventricular posterior wall is not clear. ${ }^{5)}$ When the results of Tan et al. ${ }^{5)}$ on the diameter ratio of the CA to the aortic valve annulus were compared with the findings of the current study according to the pattern of coronary dominance, significant differences were found in both right and left dominance. The comparison with the study of Choi et al. ${ }^{4)}$ showed that in left coronary dominance, the the studies have significant differences in the diameter ratio of the LCA to the RCA. Choi et al. ${ }^{4)}$ and Tan et al. ${ }^{5}$ did not consider coronary dominance, and the comparisons between their results and the current study's findings suggest that the accuracy of CAL diagnosis in children with left coronary dominance may decrease when coronary dominance is not taken into account.

CA indices were significantly different according to the pattern of coronary dominance in the present study. Therefore, to assess CAL abnormality in children, the pattern of coronary dominance should first be determined, and CA indices can be useful in determining the coronary dominance.

It is difficult to determine coronary dominance with echocardiography as accurately as with CAG or CCTA. Owing to the small sample size, data were not analyzed according to sex or age. In conclusion, coronary artery indices showed significant differences according to coronary dominance patterns in early childhood. CA indices can be useful in determining the coronary dominance.

\section{Conflicts of interest}

No potential conflict of interest relevant to this article was reported.

\section{References}

1. Baraona F, Valente AM, Porayette P, Pluchinotta FR, Sanders SP. Coronary arteries in childhood heart disease: implications for management of young adults. J Clin Exp Cardiolog 2012;(Suppl 8). pii: 006.

2. JCS Joint Working Group. Guidelines for diagnosis and management of cardiovascular sequelae in Kawasaki disease (JCS 2013). Digest version. Circ J 2014;78:2521-62.

3. McCrindle BW, Rowley AH, Newburger JW, Burns JC, Bolger AF, Gewitz M, et al. Diagnosis, treatment, and long-term management of Kawasaki disease: a scientific statement for health professionals from the American Heart Association. Circulation 2017;135:e927-99.

4. Choi JY, Yun YS, Noh CI, Choi JU, Kang IS. Dimension of normal coronary arteries determined by cross-sectional echocardiography. J Korean Pediatr Soc 1992;35:1336-42.

5. Tan TH, Wong KY, Cheng TK, Heng JT. Coronary normograms and the coronary-aorta index: objective determinants of coronary artery dilatation. Pediatr Cardiol 2003;24:328-35.

6. Fuse S, Kobayashi T, Arakaki Y, Ogawa S, Katoh H, Sakamoto N, et al. Standard method for ultratsound imaging of coronary artery in children. Pediatr Int 2010;52:876-82.

7. Capannari TE, Daniels SR, Meyer RA, Schwartz DC, Kaplan S. Sensitivity, specificity and predictive value of two-dimensional echocardiography in detecting coronary artery aneurysms in patients with Kawasaki disease. J Am Coll Cardiol 1986;7:355-60.

8. Arjunan K, Daniels SR, Meyer RA, Schwartz DC, Barron H, Kaplan S. Coronary artery caliber in normal children and patients with Kawasaki disease but without aneurysms: an echocardiographic and angiographic study. J Am Coll Cardiol 1986;8:1119-24.

9. de Zorzi A, Colan SD, Gauvreau K, Baker AL, Sundel RP, Newburger JW. Coronary artery dimensions may be misclassified as normal in Kawasaki disease. J Pediatr 1998;133:254-8.

10. Dallaire F, Dahdah N. New equations and a critical appraisal of coronary artery Z scores in healthy children. J Am Soc Echocardiogr 2011; 24:60-74.

11. Vegsundvåg J, Holte E, Wiseth R, Hegbom K, Hole T. Transthoracic echocardiography for imaging of the different coronary artery segments: a feasibility study. Cardiovasc Ultrasound 2009;7:58.

12. Shriki JE, Shinbane JS, Rashid MA, Hindoyan A, Withey JG, DeFrance A, et al. Identifying, characterizing, and classifying congenital anomalies of the coronary arteries. Radiographics 2012;32:453-68.

13. Gabella G. Cardiovascular. In: Williams PL, Bannister LH, Berry MM, editors. Gray's anatomy. 38th ed. London: Churchill Livingstone, 1995:1451-576.

14. Angelini P. Coronary artery anomalies--current clinical issues: definitions, classification, incidence, clinical relevance, and treatment guidelines. Tex Heart Inst J 2002;29:271-8.

15. Gebhard C, Fuchs TA, Stehli J, Gransar H, Berman DS, Budoff MJ, et al. Coronary dominance and prognosis in patients undergoing coronary computed tomographic angiography: results from the CONFIRM (COronary CT Angiography EvaluatioN For Clinical Outcomes: An InteRnational Multicenter) registry. Eur Heart J Cardiovasc Imaging 2015;16:853-62.

16. Veltman CE, de Graaf FR, Schuijf JD, van Werkhoven JM, Jukema JW, Kaufmann PA, et al. Prognostic value of coronary vessel dominance in relation to significant coronary artery disease determined with non-invasive computed tomography coronary angiography. Eur Heart J 2012;33:1367-77.

17. Dodge JT Jr, Brown BG, Bolson EL, Dodge HT. Lumen diameter of normal human coronary arteries. Influence of age, sex, anatomic variation, and left ventricular hypertrophy or dilation. Circulation 1992; 86:232-46. 